\title{
Nurses and Midwives Are on the COVID-19 Frontline: Spiritual Care
}

\section{Matters}

\author{
Fereshteh Narenji ${ }^{1}$ and Nazi Nejat ${ }^{2,}{ }^{*}$ \\ ${ }^{1}$ School of Medicine, Arak University of Medical Sciences, Arak, Iran \\ ${ }^{2}$ School of Nursing, Arak University of Medical Sciences, Arak, Iran \\ "Corresponding author: School of Nursing, Arak University of Medical Sciences, Arak, Iran. Tel:+98-9124396392, Email: n.nejat@arakmu.ac.ir
}

Received 2020 August 28; Revised 2020 September 21; Accepted 2020 October 25.

Keywords: Nurse, Midwive, COVID-19, Spiritual Care

\section{Dear editor,}

Nurses and midwives are at higher risk of various infections than the general population because they are in direct contact with patients (1). This issue is considered in their education tutorials, with special emphasis on selfcare (2). The recent pandemic has significantly increased the risk of both infection and mortality among health staff, especially nurses and midwives. Severe acute respiratory syndrome coronavirus 2 is a highly contagious and rapidly growing virus that carries high mortality with no drug or vaccine. The workload of nurses and midwives who are at the frontline of coping with Covid-19 has increased significantly so that they do not have enough time for their daily activities or mental well-being (3). Hence, Covid-19 is a major threat to the health and lives of nurses and midwives.

These issues, along with the high mortality of patients, have caused great emotional problems for nurses and midwives when dealing with patients. Although many people suffer from varying degrees of psychological disorders (4), nurses and midwives are experiencing an increase in psychological pressure. Most of the nurses and midwives dealing with the Covid-19 virus are experiencing increased levels of anxiety and may experience a cycle of fear and difficulty in coping with the problem. Anger, emotional problems, focusing on coping with the stress of Covid-19, anxiety, and sadness are reported by Huang et al. (2020) in a study on nurses working at Covid-19 departments (5).

Therefore, it has been recommended to support nurses and midwives emotionally through effective coping strategies, to provide a strong guarantee for their decisive victory in the battle to prevent and control epidemics, providing in-time support and psychological assistance, and training them about how to deal with crises. Paying special attention to spiritual health is one of the best psy- chological supports that can be provided. Several studies mentioned training nurses on providing spiritual care to patients, especially those with an incurable disease or for end-stage care, however, enough attention is not paid to the spiritual health of nurses and midwives during the Covid-19 pandemic. Although several factors contribute to the spiritual needs of individuals but cultural backgrounds also have an important role in assessing and addressing the needs of formal caregivers, including nurses and midwives. Taylor (2006) believed that spirituality contains three components: intrapersonal, interpersonal, and transpersonal. Examples of transcendental spiritual needs include the desire to communicate with and worship a superior being (God), while the desire for forgiveness, love, and affection reflects interpersonal, spiritual needs (6).

Spiritual care can be very effective in crises that disrupt the individual's belief system and values and ultimately affect all aspects of human existence (physical, mental, psychological, and social). Organizing values, relationships, meaning, and purpose of life as well as increasing the spiritual health contribute to our well-being. Creating a sense of personal growth and, as a result, coping with the crisis evokes positive emotions such as hope and satisfaction, optimism, a sense of inner peace, and the ability to enjoy life.

Training and providing spiritual care to nurses and midwives based on the needs emphasized by the Fisher model (1998) (i.e., individual, social, environmental, and transcendental dimensions (belief in a superpower, need to communicate with others, internal coordination, maintaining one's existential integrity and Strong support systems, nature care and a sense of connection with the world around them using individual methods of acquiring and promoting spirituality, such as mindfulness methods, prayer, yoga, etc.) can reduce the physical and emo- 
tional stress-related to caring from patients, promoting nurses and midwives' health status, and, consequently, improving the quality of service provided to patients (7). Therefore, community health managers and policymakers should increase the spiritual health of medical staff by continuously assessing their spiritual needs and implementing appropriate programs.

\section{Footnotes}

Authors' Contribution: Developed the original idea and the protocol N. N. wrote the manuscript, N.N. and F.N. prepared the manuscript N.N and F.N.

Conflict of Interests: The authors declare no conflicts of interest.

Funding/Support: None.

\section{References}

1. Baldacchino DR. Teaching on spiritual care: The perceived impact on qualified nurses. Nurse Educ Pract. 2011;11(1):47-53. doi: 10.1016/j.nepr.2010.06.008.

2. Bernard H, Fischer R, Mikolajczyk RT, Kretzschmar M, Wildner M. Nurses' contacts and potential for infectious disease transmission. Emerg Infect Dis. 2009;15(9):1438-44. doi:10.3201/eid1509.081475.

3. United Nation News. COVID-19 highlights nurses' vulnerability as backbone to health services worldwide. 2020, [cited 25 Aug 2020]. Available from: https://news.un.org/en/story/2020/04/1061232.

4. World Health Organization. Mental health and psychosocial considerations during the COVID-19 outbreak, 18 March 2020. WHO; 2020.

5. Huang L, xu FM, Liu HR. Emotional responses and coping strategies of nurses and nursing college students during COVID-19 outbreak. MedRxiv. 2020. doi: 10.1101/2020.03.05.20031898.

6. Taylor EJ. Prevalence and Associated Factors of Spiritual Needs Among Patients With Cancer and Family Caregivers. Oncol Nurs Forum. 2006;33(4):729-35. doi:10.1188/06.Onf.729-735.

7. Fisher J. Development and application of a spiritual well-being questionnaire called SHALOM. Religions. 2010;1(1):105-21. doi: $10.3390 /$ rel1010105 